\title{
Comparative study of different histologic classifications in the degree of differentiation in endometrial adenocarcinoma
}

\author{
Agnaldo L. Silva-Filho ${ }^{1,2}$, Érica B.S. Xavier ${ }^{3}$, Eduardo B. Cândido ${ }^{1}$, Ricardo Macarenco ${ }^{4}$, Márcia C.F. Ferreira ${ }^{1}$, \\ Marcelo A.P. Xavier ${ }^{5}$, Rayra A.M. Maciel $^{6}$, Paula V.T. Vidigal ${ }^{5}$ \\ ${ }^{1}$ Department of Obstetrics and Gynecology, Federal University of Minas Gerais (UFMG), Belo Horizonte, MG - Brazil \\ ${ }^{2}$ Department of Obstetrics, Gynecology and Mastology, Botucatu Medical School, UNESP-São Paulo State University, Botucatu, SP - Brazil \\ ${ }^{3}$ Clinical Hospital of Medical School of Federal University of Minas Gerais, Belo Horizonte, MG - Brazil \\ ${ }^{4}$ Department of Pathology, Botucatu Medical School, UNESP-São Paulo State University, Botucatu, SP - Brazil \\ ${ }^{5}$ Department of Pathology, Federal University of Minas Gerais (UFMG), Belo Horizonte, MG - Brazil \\ ${ }^{6}$ Academic of Medical School of Federal University of Minas Gerais, Belo Horizonte, MG - Brazil
}

\begin{abstract}
Purpose: To evaluate the concordance among the available histologic classifications for endometrial adenocarcinoma using interobserver and intraobserver agreement as well as the association of tumor histologic degree in the above mentioned classifications with cellular proliferation measured by Ki- 67.

Methods: Seventy women who underwent surgical treatment of endometrial adenocarcinoma with histologic confirmation of endometrioid type were included in the study. Two experienced pathologists randomly analyzed the slides in 3 distinct timeframes with a maximum of 25 slides/timeframe. Tumor slides were classified according to the degree of differentiation using 4 different classifications: International Federation of Gynecology and Obstetrics (FIGO), modified FIGO, Lax, and Alkushi.

Results: Intraobserver agreement was reasonable for classification of FIGO (k 0.469 and 0.538 ), very good for modified FIGO (k 0.661 and 0.768), moderate for Lax classification ( 0.496 and 0.466 ), and moderate/good for Alkushi classification ( 0.528 and 0.736 ). Interobserver concordance was regular for FIGO classification ( $\mathrm{k}=0.271$ and 0.343 ), good/moderate for modified FIGO classification ( $k=0.661$ and 0.522 , respectively), regular/moderate for Lax classification ( $k=0.258$ and 0.465 , respectively), and regular for Alkushi classification ( $k=0.283$ and 0.402 ).

Conclusions: The prognostic value of histologic grading in endometrial carcinoma and its importance for a successful therapeutic plan have been documented repeatedly, but the best grading system, in terms of prognostication, reproducibility, ease of use, and universality (e.g., applicability to all tumor cell types), has not been unequivocally defined.
\end{abstract}

Keywords: Differential diagnosis, Endometrial carcinoma, Immunohistochemistry

\section{Introduction}

Endometrial cancer is the most common cancer of the female genital tract $(1,2)$. Most patients are diagnosed with early-stage disease and therefore have an excellent 5-year

\footnotetext{
Accepted: April 22, 2016

Published online: August 2, 2016

Corresponding author:

Agnaldo L. Silva-Filho, MD, PhD

Department of Obstetrics and Gynecology

Federal University of Minas Gerais (UFMG)

Avenida Professor Alfredo Balena 190

30130100 Santa Efigênia

Belo Horizonte, Minas Gerais, Brazil

agnaldo.ufmg@gmail.com
}

survival rate, of approximately $85 \%$ (2). The pathologic stage of the disease, the extent of myometrial invasion, the presence of lymphovascular invasion, and the histologic subtype are considered to have prognostic value for patients with endometrial carcinoma $(3,4)$. Factors such as histologic type, histologic grade, myometrial infiltration, peritoneal cytology, adnexal and lymph node metastases, and lymphovascular space invasion (5-8) are usually described to predict the behavior of endometrial carcinomas. Most cancers of the endometrium are of endometrioid histology, followed by serous and clear cell types $(9,10)$.

Histopathologic assessment of tumor grade and cell type is of paramount importance for the management of endometrial carcinoma, guiding the extent of surgery and the use of adjuvant radiation therapy and chemotherapy (11). The current International Federation of Gynecology and Obstetrics (FIGO) grading system of endometrial carcinomas is widely 
TABLE I - Comparative data reflect the tumor differentiation in the 4 distinct classifications proposed

\begin{tabular}{ll}
\hline Classification & Description \\
\hline FIGO & $\begin{array}{l}\text { Adenocarcinoma well-differentiated (G1): tumors that exhibit growth pattern, solid nonsquamous area corresponding } \\
\text { to }<5 \% \text { of the neoplasm; G2, a nonsquamous, nonmorula solid growth pattern comprising } 5 \%-50 \% \text { of the tumor; } \mathrm{G} 3, \text { a } \\
\text { nonsquamous, nonmorula solid growth pattern comprising }>50 \% \text { of the tumor }\end{array}$ \\
Modified FIGO & Low grade: endometrioid adenocarcinomas $\mathrm{G} 1$ and $\mathrm{G} 2$; high grade: endometrioid adenocarcinomas $\mathrm{G} 3$ \\
Lax & Low grade: presence of solid growth pattern (squamous or otherwise) of more than $50 \%$ of the tumor, or tumor necro- \\
& sis, or myometrial infiltrative growth; high grade: should have at least 2 of the 3 histologic criteria listed in low grade \\
Alkushi & Low grade: growth pattern predominantly papillary or solid, or mitotic index $\geq 6$ mitosis per 10 high power microscopic \\
& fields, or severe nuclear atypia; high grade: growth pattern predominantly papillary or solid, or mitotic index $\geq 6$ mitoses \\
& per 10 high power microscopic fields, or severe nuclear atypia
\end{tabular}

FIGO = International Federation of Gynecology and Obstetrics.

used worldwide but is not without flaws. It requires evaluation of histologic features that can be difficult to assess, including recognition of small amounts of solid growth, distinction of squamous from nonsquamous solid growth, and assessment of degree of nuclear atypia (12).

Although the importance of the histologic grade of endometrial carcinoma has been extensively documented for treatment planning and prognosis, the best grading system, considering its reproducibility, ease of use, and applicability to all tumor cell types, has not been unequivocally defined (13). In 1998, FIGO presented a revised classification still based on tumor architecture as the main grading parameter. Later, several studies confirmed the prognostic value of architectural grading and showed it to present a fair to acceptable level of reproducibility.

In an attempt to improve the prognostic value of tumor grade, several investigators have in recent years proposed a host of new grading systems, some of which are based on novel parameters (12-14). Lax et al (12) described a binary architectural grading system for uterine endometrial endometrioid carcinoma and analyzed its performance for predicting prognosis and with respect to intraobserver and interobserver reproducibility. They concluded that this binary grading system had advantages over FIGO and nuclear grading and allowed greater interobserver and intraobserver reproducibility. Their results, however, should be revalidated. Others have aimed to evaluate the interobserver reproducibility of FIGO, the architectural binary and nuclear grading systems, and correlate these grading systems with pathologic prognostic factors for endometrial endometrioid carcinoma. Apparently, binary high grade and vascular involvement are adverse prognostic factors on recurrence-free survival (15). However, the number of cases studied remains low, so no definite conclusion can be drawn.

Alkushi et al (13) used 3 grading systems: FIGO grading, the binary grading system proposed by Lax et al (12), and a new grading system based on tumor architecture, nuclear atypia, and mitotic index. They combined grades 1 and 2 of the FIGO grading system, which resulted in a highly reproducible 2-tiered system, with a degree of reproducibility similar to that of the other 2-tiered systems tested and without loss of prognostic power $(12,13)$ (Tab. I).

Immunohistochemistry has been increasingly used as a diagnostic aid in differential diagnosis of endometrioid and serous carcinoma. Immunomarkers have been introduced in clinical research, but so far no single marker is completely sensitive or specific for a given cell type, so a panel of markers may be required (11). Ki-67 is a large nucleolar phosphoprotein expressed in tight association with the cell cycle. It is strictly associated with cell proliferation (16), and has increasingly been used as a target in the treatment of various types of cancer $(17,18)$.

The aim of this study was to evaluate the agreement among the available histologic grading classifications (FIGO, modified FIGO, Lax, and Alkushi) $(9,12,13,19)$ of endometrial adenocarcinoma using interobserver and intraobserver agreement as well as the association of tumor histologic grading in the above mentioned classifications with cellular proliferation measured by Ki-67 in women with endometrial adenocarcinoma.

\section{Methods}

Seventy women who underwent surgical treatment of endometrial adenocarcinoma with histologic confirmation of endometriod type were included in the study. Other inclusion criteria were the absence of previous chemotherapy or radiotherapy and the availability of good-quality paraffinembedded specimens for histopathologic analysis. All histopathologic analyses were performed at CIPAX Laboratory at São José dos Campos (SP) city. The study protocol was approved by the Ethics Committee of Universidade Federal de Minas Gerais (UFMG) as well as CIPAX Laboratory. A written informed consent from all patients was also obtained. Endometrial adenocarcinoma staging was done in accordance with FIGO recommendations (9).

Two experienced pathologists with extensive training in gynecologic oncology performed the histologic evaluation. All slides were sequentially numbered and both pathologists were blind to any patient data (clinical or histologic diagnosis). Patient data were registered in a separate sheet.

\section{Histologic evaluation}

Slides stained with the hematoxylin \& eosin technique were classified using the histologic grade, reflecting the tumor differentiation, in the 4 distinct classifications 


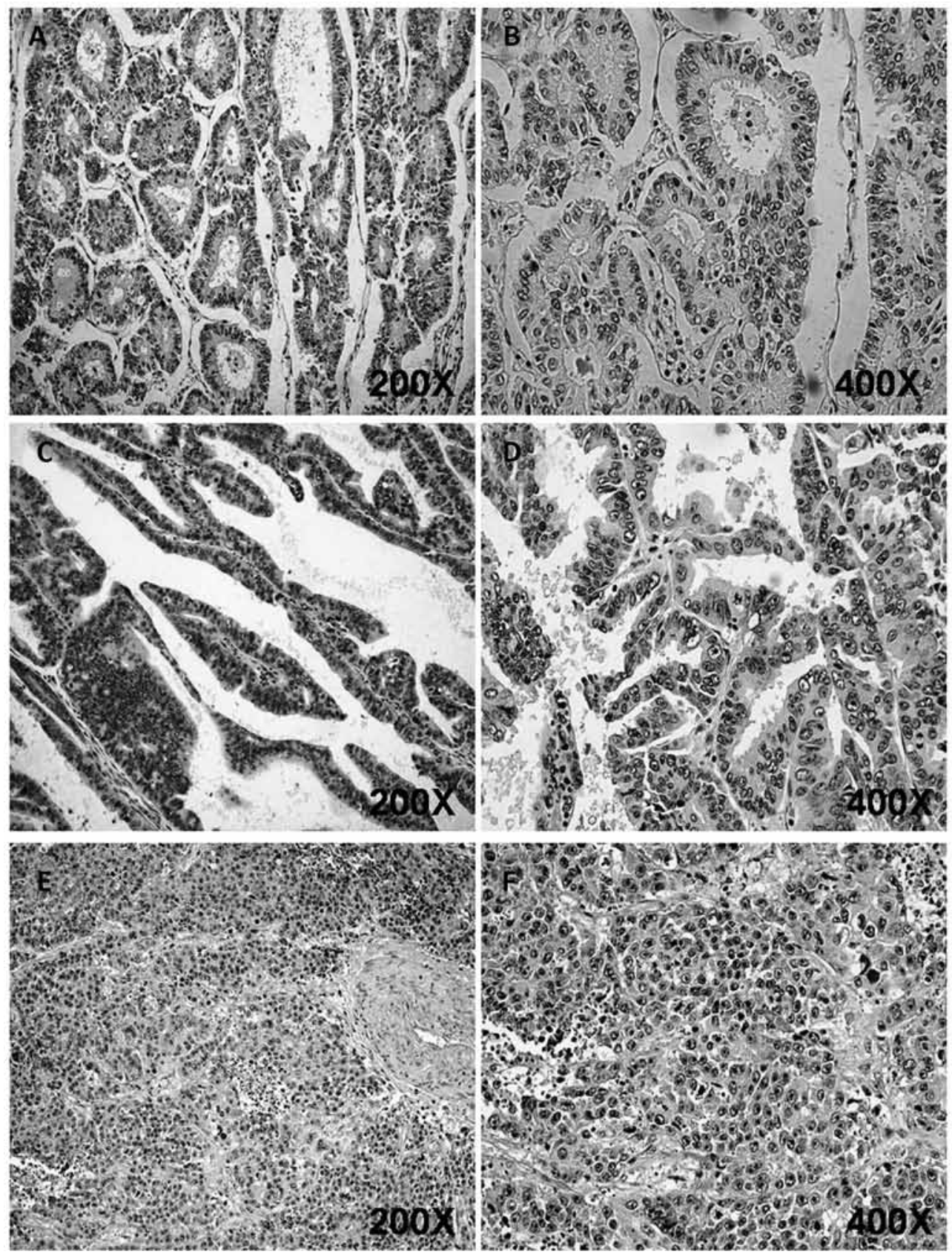

Fig. 1 - Photomicrography slides with magnifications 200× (A, C, E) and $400 \times(B, D, F)$ show the histopathologic classifications of adenocarcinoma: $\mathrm{G} 1$ ( $\mathbf{A}$ and $\mathbf{B}), \mathrm{G} 2$ (C and $\mathbf{D})$, and G3 (E and F). G1: well-differentiated tumor; G2: moderately differentiated tumor; G3: undifferentiated tumor.

proposed (FIGO, FIGO modified, Lax, and Alkushi), as shown in Figure $1(9,12,13,19)$. Two pathologists randomly analyzed the slides in 3 distinct timeframes with a maximum of 25 slides by timeframe. Interobserver agreement was obtained comparing the results of the evaluation made by the 2 pathologists.

After a minimum period of 2 months, slides were independently evaluated once more by the same pathologists. They remained blinded to each other's evaluation as well as to their own previous report. To access intraobserver agreement, the results of the first evaluation were compared to those obtained in the second analysis by the same professional.

\section{Immunohistochemistry}

Cellular proliferation index, measured by Ki-67 expression, was assessed by immunohistochemistry using the monoclonal antibody MIB-1 (1:100) against the nuclear antigen found in the proliferation phase (clone MIB-1, product number M7240, Dako Corp., Glostrup, Denmark).

Immunohistochemistry was performed by the avidinbiotin-peroxidase technique using Vectastain ${ }^{\circledR}$ Universal Elite ABC kit (Vector Laboratories, Burlingame, CA, USA). Samples were fixed with a $10 \%$ paraformaldehyde solution, embedded in paraffin, and cut into $4-\mu \mathrm{m}$ sections. The sections 


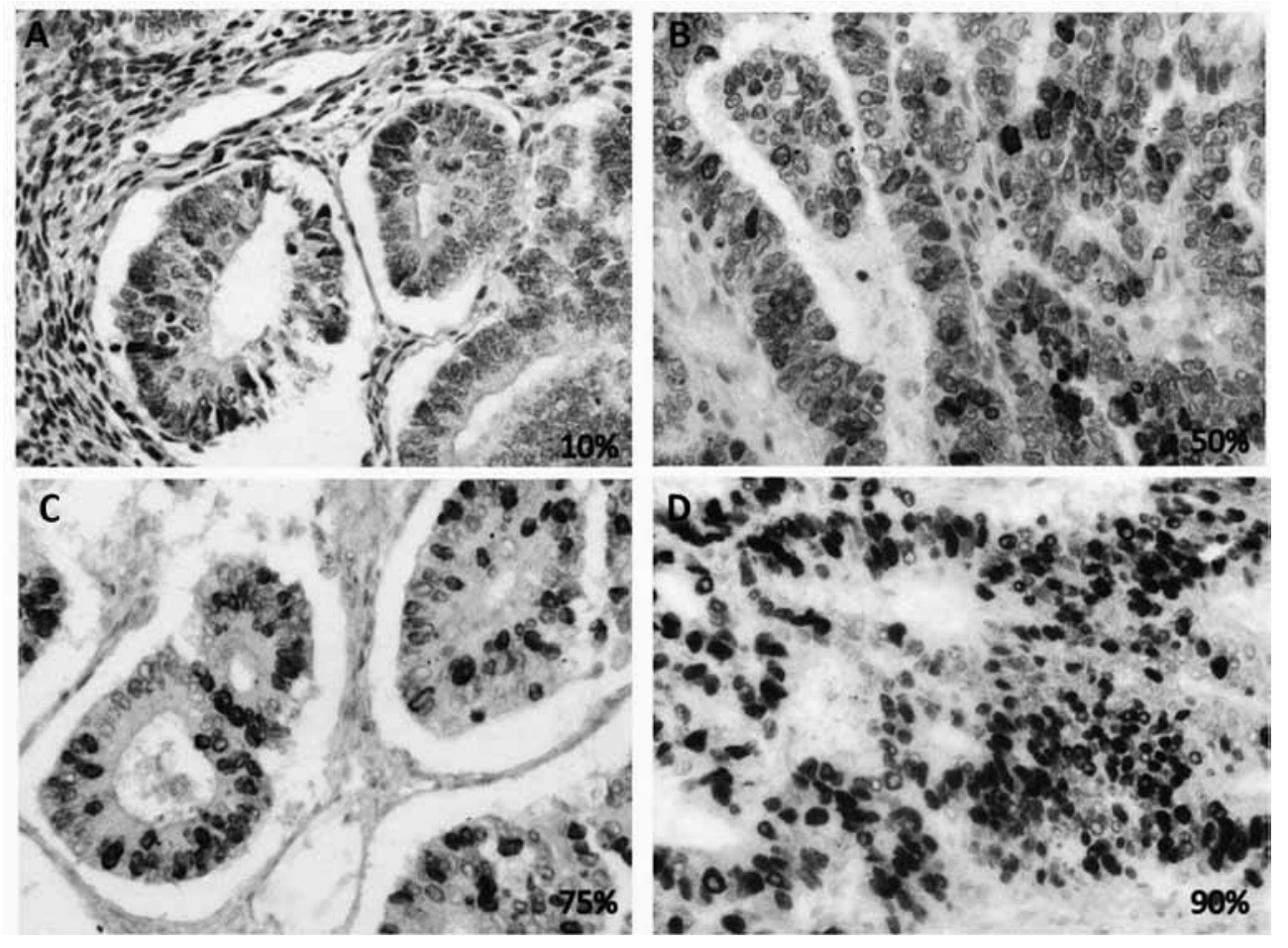

Fig. 2 - Immunohistochemistry analysis shows cellular proliferation index, measured by Ki-67 marker expression. Each preparation was evaluated in the same optical microscope at 200x. Quantitative analysis of nuclear atypia in tumor cells was performed and the results registered as percentages ranging from $5 \%$ to $90 \%$ : A < $10 \%$; B $10 \%-50 \%$; C 51\%-75\%; D: 76\%-90\%. were then deparaffinized and hydrated in decreasing concentrations of ethanol, washed in phosphate-buffered saline (PBS), and incubated with hydrogen peroxide in methanol for 30 minutes in order to block endogenous peroxidase. Following a wash in PBS, normal goat serum (Vector Laboratories) diluted 1:30 in PBS was incubated with the sections for 30 minutes at room temperature.

Each preparation was evaluated in the same optical microscope with $200 \times$ magnification by one pathologist blind to the previous histologic grade classification to reduce bias. Patients whose slides were not suitable for microscope histologic analysis were excluded from the study. Quantitative analysis of tumor cells nuclear staining was performed and results registered as percentages ranging from $5 \%$ to $90 \%$ according to Figure 2.

\section{Statistical analysis}

Data were transferred to an Excel spreadsheet (Microsoft, Redmond, WA, USA), precodified in numbers and analyzed by using SPSS 10.0 (Statistical Package for the Social Sciences; SPSS, Inc., Chicago, IL, USA). Quantification of interobserver and intraobserver variations was obtained by calculating the kappa index, which showed 5 distinct possibilities: excellent (0.81-1.00), very good (0.61-0.80), moderate (0.41-0.60), regular (0.21-0.40), and weak (0.01-0.20).

Group differences were evaluated using chi-square test (endometrial cancer staging), Student t, or analysis of variance (cellular proliferation). Differences were considered statistically significant at $p<0.05$.

A unique score of histologic grade for each classification (final grading) was created, based on the results of the 4 analyzes made by the 2 pathologists. When at least 3 results agreed, the respective grade was reported. When all evaluations were discordant or only 2 results were in agreement, data were reported as no agreement. These final grading data were correlated with surgical FIGO staging using the chi-square test.

\section{Results}

The age of the patients ranged from 30 to 86 years (mean 62.92 years). The clinical and surgical staging (FIGO) IA was the most common, present in $42(60 \%)$ patients. Stage IB was found in 19 patients (27.1\%), IIA in $3(4.3 \%)$, IIB in 1 (1.4\%), IIIA in 4 (5.7\%), and IIIC in 1 woman (1.4\%).

Intraobserver agreement was moderate for FIGO classification ( $k=0.503)$, very good for modified FIGO classification ( $k=0.714)$, moderate for Lax classification $(k=0.481)$, and very good for Alkushi classification ( $k=0.632)$. Interobserver evaluations showed the following results: regular for FIGO classification ( $k=0.307)$, moderate for modified FIGO ( $k=0.591)$, regular for Lax classification $(k=0.361)$, and regular for Alkushi classification ( $k=0.342$ ) (Tabs. II and III). No association was found between the histologic classifications FIGO ( $p=0.663)$, modified FIGO ( $p=0.370)$, Lax $(p=0.402)$, and Alkushi ( $p=0.101$ ) with tumor staging (Tab. IV).

There was an association of the histologic classifications FIGO ( $p=0.039)$, modified FIGO ( $p=0.017)$, and Alkushi ( $p=0.039$ ) with Ki-67 expression (Fig. 3).

As for reproducibility of the 3 classifications, the modified FIGO classification showed the highest degree of agreement.

\section{Discussion}

Since 1930, gynecologists who deal with women's tumors have tried to stablish precise criteria to improve therapeutic 
TABLE II - Kappa indexes for intraobserver and interobserver agreement in each histologic classification

\begin{tabular}{|c|c|c|c|c|c|c|}
\hline & \multicolumn{3}{|c|}{ Intraobserver } & \multicolumn{3}{|c|}{ Interobserver } \\
\hline FIGO & 0.469 & 0.538 & 0.503 & 0.271 & 0.343 & 0.307 \\
\hline Modified FIGO & 0.661 & 0.768 & 0.714 & 0.661 & 0.522 & 0.591 \\
\hline Lax & 0.496 & 0.466 & 0.481 & 0.258 & 0.465 & 0.361 \\
\hline
\end{tabular}

FIGO = International Federation of Gynecology and Obstetrics.

TABLE III - Absolute and percentage values for each category of histologic classifications for endometrial adenocarcinoma

\begin{tabular}{llcc}
\hline Classification & Category & No. & Percentage \\
\hline FIGO & No agreement & 20 & 28.6 \\
& Grade 1 & 25 & 35.7 \\
& Grade 2 & 13 & 18.6 \\
& Grade 3 & 12 & 17.1 \\
Modified FIGO & No agreement & 6 & 8.6 \\
& Low grade & 52 & 74.3 \\
& High grade & 12 & 17.1 \\
Lax & No agreement & 9 & 12.9 \\
& Low grade & 51 & 72.9 \\
Alkushi & High grade & 10 & 14.3 \\
& No agreement & 12 & 17.1 \\
& Low grade & 40 & 57.1 \\
& High grade & 18 & 25.7 \\
\hline
\end{tabular}

FIGO = International Federation of Gynecology and Obstetrics.
TABLE IV - Correlation between histologic classification and clinical staging

\begin{tabular}{lccc}
\hline Classification & \multicolumn{2}{c}{ Staging, $\mathbf{n}$ (\%) } & p \\
\cline { 2 - 3 } & \multicolumn{1}{c}{ IA } & IB & 0.663 \\
FIGO & & & \\
G1 & $16(55.2)$ & $9(45.0)$ & \\
G2 & $7(24.1)$ & $5(25.0)$ & \\
G3 & $6(20.7)$ & $6(30.0)$ & \\
Modified FIGO & & & 0.370 \\
$\quad$ Low grade & $31(83.8)$ & $21(77.8)$ & \\
High grade & $6(16.2)$ & $6(22.2)$ & \\
Lax & & & \\
Low grade & $31(86.1)$ & $20(80)$ & \\
High grade & $5(13.9)$ & $5(20)$ & \\
Alkushi & & & \\
Low grade & $24(70.6)$ & $16(66.7)$ & \\
High grade & $10(29.4)$ & $8(33.3)$ & \\
\hline
\end{tabular}

$\mathrm{FIGO}=$ International Federation of Gynecology and Obstetrics.
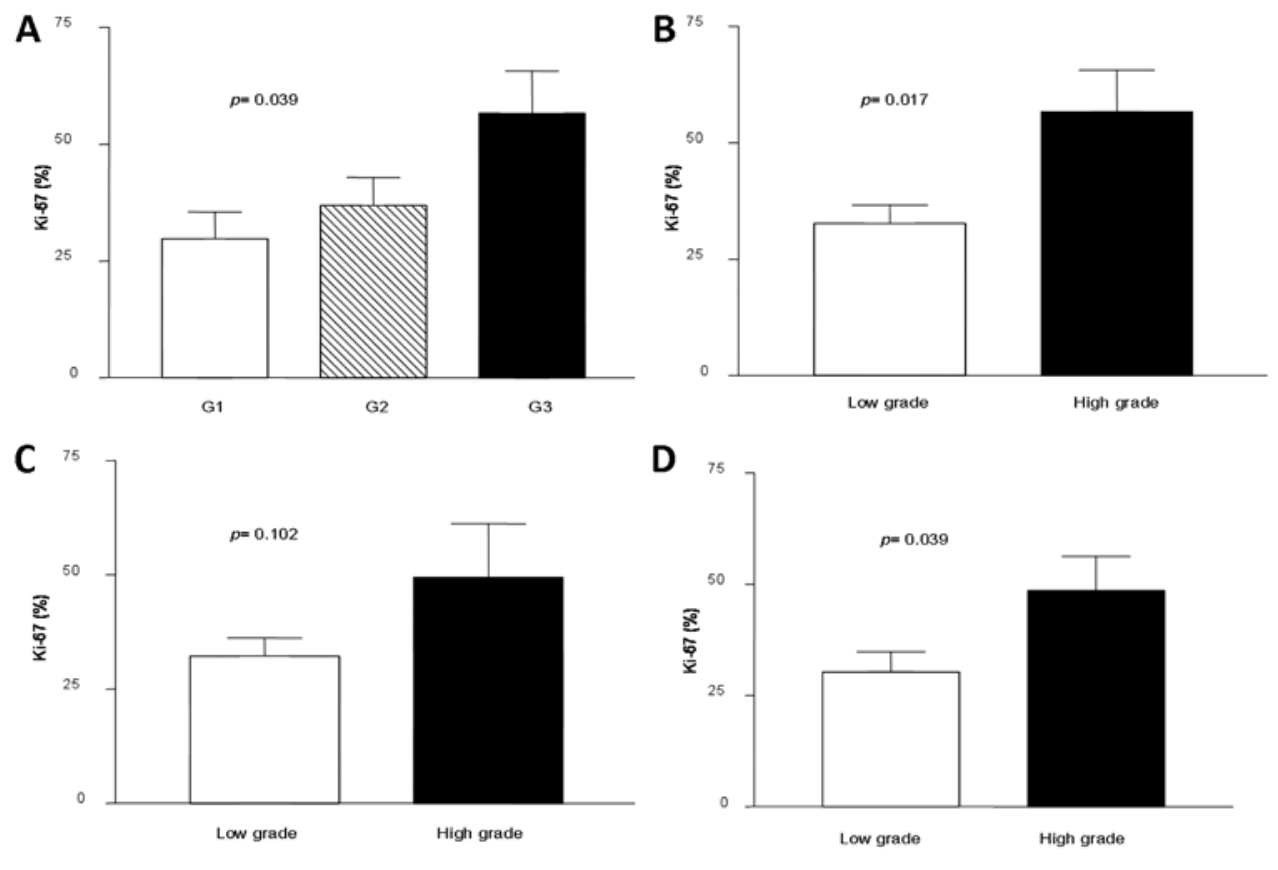

Fig. 3 - Association between Ki-67 protein expression and the degree of cell differentiation in International Federation of Gynecology and Obstetrics (FIGO) (A), modified FIGO (B), Lax (C), and Alkushi (D) classifications in endometrial adenocarcinoma. The $p$ values were calculated by analysis of variance and Student $t$ test. Only nuclear staining was seen in the quantification of the protein $\mathrm{Ki}-67$. 
TABLE V - Studies evaluating the 4 classifications and nuclear grade differentiation comparing the results with the findings in the current study

\begin{tabular}{|c|c|c|c|c|c|c|}
\hline Classification & FIGO & Modified FIGO & Lax & Alkushi & Nuclear grade & No. \\
\hline \multicolumn{7}{|c|}{ Interobserver concordance (kappa) } \\
\hline Lax et al (12) & 0.55 & NA & 0.65 & NA & 0.22 & 141 \\
\hline Stefansson et al (27) & 0.54 & NA & 0.63 & NA & NA & 30 \\
\hline Scholten et al (21) & 0.41 & 0.58 & 0.39 & NA & NA & 800 \\
\hline Sagae et al (22) & 0.50 & NA & 0.57 & NA & 0.23 & 200 \\
\hline Alkushi et al (13) & 0.61 & 0.71 & 0.75 & 0.76 & NA & 202 \\
\hline Kapucuoglu et al (15) & 0.67 & NA & 0.65 & NA & 0.09 & 88 \\
\hline Xavier et al (present study) & 0.30 & 0.59 & 0.36 & 0.34 & NA & 70 \\
\hline \multicolumn{7}{|c|}{ Intraobserver Concordance (kappa) } \\
\hline Lax et al (12) & 0.67 & NA & 0.79 & NA & 0.41 & 141 \\
\hline Sagae et al (22) & 0.62 & NA & 0.62 & NA & 0.43 & 200 \\
\hline Alkushi et al (13) & 0.73 & 0.90 & 0.75 & 0.80 & NA & 202 \\
\hline Xavier et al (present study) & 0.50 & 0.71 & 0.48 & 0.63 & NA & 70 \\
\hline
\end{tabular}

FIGO = International Federation of Gynecology and Obstetrics.

management of oncologic patients. The goal is to obtain a uniform terminology and a grading system capable of providing accurate prognosis and enhance communication among the professionals involved in the treatment course (19).

Our study evaluated histologic classifications of the differentiation grades of endometrial adenocarcinoma. These classifications assume great importance in clinical management, guiding surgical interventions and adjuvant treatments, such as chemotherapy and radiotherapy. Therefore, histologic assessment of differentiation grade has prognostic and therapeutic implications. However, this assessment is usually performed by one only pathologist, which makes it susceptible to subjective impressions, limiting the reliability of the result (20).

The FIGO classification is the most used and has moderate to good reproducibility. Other classification systems have been proposed in an attempt to improve the reproducibility and the prognostic ability of this histologic finding (15) (Tab. V).

We also evaluated another factor that could possibly be of value in the prognosis assessment of the endometrium adenocarcinoma. Ki-67, a nonhistone protein with short halflife, is expressed in the nuclei of proliferating cells, becoming a marker of cell proliferation. The presence of mitosis and its extent are used in some classifications, e.g., the Alkushi system, to identify tumors of less favorable biological behavior and poor prognosis. A proliferation cell marker, such as Ki-67, could be an indirect and more reliable way to access mitotic index.

The result of the histologic classifications of the differentiation grade, as any other result involving measurements, presents variations. Studies evaluating the histologic classifications of endometrioid adenocarcinomas were not able to propose a system that was not influenced by subjectivity and was easily reproducible $(15,20)$.

Limitations in the reproducibility of the classifications could interfere in clinical diagnosis, overestimating or underestimating the biological behavior of the tumor, and influencing therapeutic decisions.
Several studies evaluated intraobserver and interobserver agreement in determining this histopathologic finding (Tab. IV). Lax et al (12) proposed a binary classification of the cellular differentiation grade of endometrial adenocarcinoma. The comparison of this classification with the one from FIGO and the nuclear grade showed an intraobserver kappa index of $0.79,0.67$, and 0.41 , respectively. Interobserver kappa index was $0.65,0.55$, and 0.22 in the 3 classifications, respectively $(12,13,15)$.

An evaluation of 800 patients was done by 2 pathologists of distinct diagnostic centers, using 3 classifications: FIGO, modified FIGO, and Lax, with interobserver kappa indexes of $0.41,0.58$, and 0.39 , respectively $(20,21)$. Another study evaluated 200 cases, with histologic analysis done by 5 pathologists, and showed the following kappa indexes for interobserver agreement: 0.50 for FIGO classification, 0.57 for Lax classification, and 0.23 for nuclear grade. Intraobserver agreement was 0.62 for FIGO classification, 0.62 for Lax classification, and 0.43 for nuclear grading (22).

Demczuk et al (23) evaluated interobserver agreement in the analysis of 133 samples of patients with endometrial adenocarcinoma in 2 classifications: FIGO and Lax. Of the 5 specialists participating in the study, 3 were experienced in gynecologic pathology and the other 2 were not. Results were better in the group of experienced pathologists (FIGO: 0.64-0.71; Lax: 0.91-0.92) than in the group of inexperienced pathologists (FIGO 0.23-0.48; Lax: 0.21-0.57). This shows the importance of previous experience in the interpretation of differentiation grades (23).

Alkushi et al (13) proposed another classification in 2005. Their study evaluated intraobserver and interobserver agreement in their system, comparing FIGO, modified FIGO, and Lax classifications. The results showed a kappa index for interobserver agreement of 0.76 , better than all the other 4 classifications, although very close to the value obtained for Lax classification (kappa 0.75). Intraobserver agreement was greater than interobserver in all 4 systems: 
Alkushi: 0.80, FIGO: 0.73, modified FIGO: 0.90, and Lax: 0.75 (13).

In the present study, modified FIGO binary classification showed the best intraobserver and interobserver agreement. Thus, the substitution of the FIGO traditional classification of differentiation grade by the binary system could result in better reproducibility (21). Other studies presented similar results and considered the binary systems less ambiguous, because there are no intermediate classification grades. Since the prognosis does not differ between grades 1 and 2, the binary systems could be more advantageous than the FIGO one, the most used currently (20).

Our kappa indexes for intraobserver and interobserver agreement were, in general, lower than the ones obtained in other studies (Tab. IV).

The FIGO oncologic gynecology committee considers the determination of cell differentiation grade as a part of endometrial cancer staging and as an independent prognostic factor. We did not find any association of the cellular differentiation grade with FIGO tumor staging, suggesting that they may be independent factors in prognosis.

Our study found an association of a marker of cell proliferation-Ki-67-with the histologic differentiation grade in 3 of the 4 classifications evaluated. Ki-67 expression was greater in tumors considered high grade in the cellular differentiation classifications. Other studies have found that Ki-67 expression was associated with tumor staging and histologic grading $(24,25)$. The proliferation index, accessed by Ki-67 expression, has been considered a prognostic factor. Patients with Ki-67 expression up to $35 \%$ had better survival rates than ones with greater expression percentages (26).

A retrospective study that focused on the expression of Ki-67 protein in patients with endometrioid endometrial adenocarcinoma found that it was associated with the differentiation grade. G3 tumors in FIGO classification showed an expression percentage of $28 \%$, compared to $15 \%$ in G1-2 tumors. Tumor cell proliferation estimated by Ki-67 was clearly associated with the number of mitosis found in the slides (27).

Clinical course of endometrial cancer is related to tumor proliferative activity, which can be evaluated by several ways, including Ki-67 expression. Our results are in consonance with others that confirm the importance of $\mathrm{Ki}-67$ expression as a prognosis marker.

A cohort study by Barlin et al (29) showed that surgical stage assignment, grade 1-2 vs grade 3 (a binary grading system), was a significant predictor of overall survival in classification and regression analysis. The landmark trial by Creasman et al (28) established grade in combination with myometrial invasion as risk factors for nodal metastasis. Notably, tumor subtype was not predictive in our classification and regression analysis, but grade and tumor subtype are intimately connected in our design, as the high-risk histologies of serous, clear cell, and carcinosarcoma are all considered grade 3 . The disease-specific and recurrence-free survivals were similar among grade 3 endometrioid endometrial carcinomas, serous carcinomas, and clear cell carcinomas. The classification and regression analysis emphasizes the importance of high grade on overall survival (29).

In the future, pathologists and gynecologists will need to practice tumor classifications together, to minimize subjectivity and improve treatment outcomes. To date, there is no excellent histologic grading system, regarding its reproducibility. New predictive factors should be evaluated to identify patients at higher risk of recurrence and smaller survival chance $(20,30)$.

Markers of angiogenesis, hormone receptors, and cell proliferation need to be better studied, along with the ones traditionally used for prognostic definition. Greater numbers of patients should be evaluated, in an attempt to obtain more reliable information on the prognostic factors and tumor activity in patients with endometrial adenocarcinoma (15).

\section{Disclosures}

Financial support: No financial support was received for this submission.

Conflict of interest: None of the authors has conflict of interest with this submission.

\section{References}

1. Jemal A, Bray F, Center MM, Ferlay J, Ward E, Forman D. Global cancer statistics. CA Cancer J Clin. 2011;61(2):69-90.

2. Rose PG. Endometrial carcinoma. N Engl J Med. 1996;335(9): 640-649.

3. Prat J. Prognostic parameters of endometrial carcinoma. Hum Pathol. 2004;35(6):649-662.

4. Abeler VM, Kjørstad KE. Endometrial adenocarcinoma in Norway. A study of a total population. Cancer. 1991;67(12): 3093-3103.

5. De Góis NM, Martins NV, Abrão FS, De Lima GR, Alves AC. Peritumorous lymph-vascular invasion, grade of histologic differentiation, and myometrial infiltration as prognostic factors of endometrial carcinoma. Rev Paul Med. 1993;111(3):385-390.

6. Sidawy MK, Silverberg SG. Endometrial carcinoma. Pathologic factors of therapeutic and prognostic significance. Pathol Annu. 1992;27(Pt 2):153-185.

7. Yura Y, Tauchi K, Koshiyama M, et al. Parametrial involvement in endometrial carcinomas: its incidence and correlation with other histological parameters. Gynecol Oncol. 1996;63(1): 114-119.

8. Zaino RJ; Gynecologic Oncology Group. Pathologic indicators of prognosis in endometrial adenocarcinoma. Selected aspects emphasizing the GOG experience. Pathol Annu. 1995;30(Pt 1):1-28.

9. Creasman WT, Odicino F, Maisonneuve P, et al. Carcinoma of the corpus uteri. FIGO $26^{\text {th }}$ Annual Report on the Results of Treatment in Gynecological Cancer. Int J Gynaecol Obstet. 2006;95(Suppl 1):S105-S143.

10. Baekelandt MM, Castiglione M, Group EGW; ESMO Guidelines Working Group. Endometrial carcinoma: ESMO clinical recommendations for diagnosis, treatment and follow-up. Ann Oncol. 2009;20(Suppl 4):29-31.

11. Clarke BA, Gilks CB. Endometrial carcinoma: controversies in histopathological assessment of grade and tumour cell type. J Clin Pathol. 2010;63(5):410-415.

12. Lax SF, Kurman RJ, Pizer ES, Wu L, Ronnett BM. A binary architectural grading system for uterine endometrial endometrioid carcinoma has superior reproducibility compared with FIGO grading and identifies subsets of advance-stage tumors with favorable and unfavorable prognosis. Am J Surg Pathol. 2000;24(9):1201-1208.

13. Alkushi A, Abdul-Rahman ZH, Lim P, et al. Description of a novel system for grading of endometrial carcinoma and 
comparison with existing grading systems. Am J Surg Pathol. 2005;29(3):295-304.

14. Guan H, Semaan A, Bandyopadhyay S, et al. Prognosis and reproducibility of new and existing binary grading systems for endometrial carcinoma compared to FIGO grading in hysterectomy specimens. Int J Gynecol Cancer. 2011;21(4): 654-660.

15. Kapucuoglu N, Bulbul D, Tulunay G, Temel MA. Reproducibility of grading systems for endometrial endometrioid carcinoma and their relation with pathologic prognostic parameters. Int J Gynecol Cancer. 2008;18(4):790-796.

16. MacCallum DE, Hall PA. Biochemical characterization of pKi67 with the identification of a mitotic-specific form associated with hyperphosphorylation and altered DNA binding. Exp Cell Res. 1999;252(1):186-198.

17. Kausch I, Lingnau A, Endl E, et al. Antisense treatment against Ki-67 mRNA inhibits proliferation and tumor growth in vitro and in vivo. Int J Cancer. 2003;105(5):710-716.

18. Zheng JN, Ma TX, Cao JY, et al. Knockdown of Ki- 67 by small interfering RNA leads to inhibition of proliferation and induction of apoptosis in human renal carcinoma cells. Life Sci. 2006;78(7):724-729.

19. Creasman W. Revised FIGO staging for carcinoma of the endometrium. Int J Gynaecol Obstet. 2009;105(2):109.

20. Scholten AN, Creutzberg CL, Noordijk EM, Smit VT. Long-term outcome in endometrial carcinoma favors a two-instead of a three-tiered grading system. Int J Radiat Oncol Biol Phys. 2002;52(4):1067-1074.

21. Scholten AN, Smit VT, Beerman H, van Putten WL, Creutzberg $\mathrm{CL}$. Prognostic significance and interobserver variability of histologic grading systems for endometrial carcinoma. Cancer. 2004;100(4):764-772.
22. Sagae $\mathrm{S}$, Saito $\mathrm{T}$, Satoh $\mathrm{M}$, et al. The reproducibility of a binary tumor grading system for uterine endometrial endometrioid carcinoma, compared with FIGO system and nuclear grading. Oncology. 2004;67(5-6):344-350.

23. Demczuk S, Wierzchowski W, Szczepański W, Dyduch G, Czopek J, Stachura J. The comparison of the agreement in determining the histological grade of uterine endometrial endometrioid carcinoma, using the three-grade FIGO classification and the two-grade system. Pol J Pathol. 2003;54(3):179-181.

24. Zhu C, Luo J, Shi H, Xie X, Ding Z. Expression of tubulin, p53, ki67, receptors for estrogen, and progesterone in endometrial cancer. Eur J Gynaecol Oncol. 2009;30(5):514-517.

25. Konstantinos K, Marios S, Anna M, Nikolaos K, Efstratios $P$, Paulina A. Expression of $\mathrm{Ki}-67$ as proliferation biomarker in imprint smears of endometrial carcinoma. Diagn Cytopathol. 2013;41(3):212-217.

26. Suthipintawong C, Wejaranayang C, Vipupinyo C. Prognostic significance of ER, PR, Ki67, c-erbB-2, and p53 in endometrial carcinoma. J Med Assoc Thai. 2008;91(12):1779-1784.

27. Stefansson IM, Salvesen HB, Immervoll H, Akslen LA. Prognostic impact of histological grade and vascular invasion compared with tumour cell proliferation in endometrial carcinoma of endometrioid type. Histopathology. 2004;44(5):472-479.

28. Creasman WT, Morrow CP, Bundy BN, Homesley HD, Graham $J E$, Heller PB. Surgical pathologic spread patterns of endometrial cancer. A Gynecologic Oncology Group Study. Cancer. 1987;60(8)(Suppl):2035-2041.

29. Barlin JN, Zhou Q, St Clair CM, et al. Classification and regression tree (CART) analysis of endometrial carcinoma: Seeing the forest for the trees. Gynecol Oncol. 2013;130(3):452-456.

30. Zaino RJ. FIGO staging of endometrial adenocarcinoma: a critical review and proposal. Int J Gynecol Pathol. 2009;28(1):1-9. 